\title{
Sensitivity and specificity of CRP as a parameter of atherosclerosis in patients with type II diabetes mellitus
}

\author{
Doaa Gaber Mizar $^{1 *}$, Hoda Abdelbadie Hussein ${ }^{1,2}$, Mohammad Abdelhadi Mashaheet ${ }^{3}$ \\ , Maher Abobakr Elamir ${ }^{1}$ \\ ${ }^{I}$ Internal Medicine Department, Faculty of Medicine, Fayoum University, Egypt \\ ${ }^{2}$ Internal medicine department, faculty of medicine, Najran University, KSA \\ ${ }^{3}$ Internal Medicine Department, Endocrinology \&Vascular Doppler Unit, Fayoum University, Egypt \\ *Corresponding author E-mail: hahussein@nu.edu.sa
}

Copyright $\odot 2014$ Mizar et. al. This is an open access article distributed under the Creative Commons Attribution License, which permits unrestricted use, distribution, and reproduction in any medium, provided the original work is properly cited.

\begin{abstract}
Background: Type 2 diabetes mellitus (T2DM) is an inflammatory atherosclerotic condition with high prevalence of cardiovascular disease. Elevated C-reactive protein (CRP) predicts cardiovascular events in T2DM patients.

Objectives: Our work aimed to assess sensitivity and specificity of CRP in diagnosis and follow up of carotid atherosclerosis in T2DM patients (detected by measuring carotid intima-media thickness CIMT).

Design and setting: It is observational cross sectional study on T2DM patients from outpatient clinic of fayoum university hospital.

Subjects and methods: Hundred patients with T2DM were included; Group I: statin un-treated, Group II: statin treated, and fifty healthy control persons. Laboratory measurement of total cholesterol, LDL, HDL, and serum CRP levels and carotid duplex scanning were done.

Results: The patients had higher levels of CRP and CIMT than control. There was strong correlation between CIMT and CRP level among group I and II. LDL in diagnosis of atherosclerosis disease illustrated sensitivity (80\%) and specificity (4\%), while HDL sensitivity (96\%) and specificity (36\%). CRP showed higher sensitivity (98\%) and higher specificity $(72 \%)$.

Conclusion: CRP level was an important parameter of atherosclerosis with good sensitivity and specificity regardless the level of LDL and HDL.
\end{abstract}

Keywords: Carotid Intima Media Thickness, C Reactive Protein, Type 2 Diabetes Mellitus.

\section{Introduction}

A number of epidemiological studies reported that increased carotid intima media thickness (CIMT) is a good predictor of future cardiovascular disease (CVD) such as myocardial infarction, stroke and death from CVD [1]. In addition, the procedure is non-invasive, inexpensive, and readily applicable and carries virtually no risk for the patient. Therefore, CIMT has now become an established surrogate marker of CVD in clinical trials to evaluate the efficacy of interventions with statins, anti-hypertensive, aspirin and anti-diabetic medications [2], [3]. Clinically evident atherosclerosis is preceded by preclinical changes in the arterial wall and inflammatory status playing a key role in atherogenesis. There is an increasing amount of evidence that high sensitivity C-reactive protein (hs-CRP) and Interleukin-6 (IL-6) are increased in individuals with CV disease, and elevated levels of these markers frequently precede the development of CV disease [4].

Statins are one of the most important medications in cardiovascular diseases since they block cholesterol synthesis by inhibiting the 3-hydroxy-3 methylglutaryl coenzyme A reductase and thus reduce low-density lipoprotein concentrations. In the last years, numerous pleiotropic properties of statins have been described, beyond their wellknown lipid lowering function. In particular, they are able to modulate inflammation, which plays a pivotal role in the atherosclerotic process. Several trials have shown a direct correlation between statin therapy and lower C-reactive protein concentrations [5]. 
In the present study, we investigated the relationship between CIMT (as a predictor of atherosclerosis) in patients with T2DM and CRP levels in these patients. In addition, the study correlated CRP levels with LDL-cholesterol levels and the use of statin therapy to evaluate which is more important predictor of atherosclerosis as regard diagnosis and statin treatment follow up.

\section{Subjects and methods}

The study included 100 patients with type 2 diabetes mellitus and 50 control of either sex. The age ranged from 40-60 years of different disease duration. They were classified into 2 groups according to statin therapy, group-I: fifty patients not receiving statin therapy, and group-II: fifty patients on statin therapy. The control group (group III) included 50 healthy, age and sex matched non-smoker subjects without history of myocardial infarction, angina, stroke, peripheral vascular disease or diabetes. The patients were subjected to full history, clinical examination, laboratory measurement of cholesterol, LDL and HDL cholesterol, blood sugar, serum CRP level and carotid duplex scanning. Informed consent was obtained from patients and controls.

The regular CRP reagent kit (latex-enhanced nephelometry) is based on an immunological reaction between CRP antisera bound to biologically inert latex particles and CRP in the test specimen. Particle-enhanced assays are based on the reaction between a soluble analyte and the corresponding antigen or antibody bound to polystyrene particles. CRP present in the test sample will form an antigen-antibody complex with the latex particles. Light scattering, measured by a nephelometric procedure after $6 \mathrm{~min}$, is proportional to the concentration of the analyte present in the sample. No special instructions such as fasting or special diets are required. Fresh or frozen human serum, heparin and EDTA plasma samples are acceptable.

Measurements of CIMT were performed on the common carotid artery. CIMT was evaluated using a high-resolution 7.5 MHz linear transducer in B mode. CIMT of the far wall was defined as the distance between the leading edge of the lumen-intima interface and the leading edge of the media-adventitia interface. CIMT was measured on each side at three sites on the longitudinal views. CIMT was calculated as the average of measurements from three different sites on each side. CIMT is generally reported in millimeters as a continuous variable with values ranging from about 0.5 to 1.0 $\mathrm{mm}$ in healthy people [6].

\section{Statistical Analysis}

- Data were collected, coded, and double entered into Microsoft Access and data analysis was performed using SPSS software version 18.

- Simple descriptive analysis in the form of numbers and percentages for qualitative data, and arithmetic means as central tendency measurement and standard deviations. For quantitative parametric data: In-depended student tTest was used to compare measures of two independent groups of quantitative data. One way ANOVA test was used in comparing more than two independent groups of quantitative data. For qualitative data: Chi square test to compare two of more than two qualitative groups.

- $\quad \mathrm{P}$ value is significant if less than 0.05

\section{Results}

A total of 100 patients with T2DM were included in this study to investigate the relation between CIMT in these patients and CRP levels, LDL, and HDL cholesterol levels.

The demographic and clinical features of the studied groups are summarized in table 1 . Their ages ranged from 40-60 years. The mean age for group I was $48.3 \pm 6$ years, group II was $49.8 \pm 6.2$ while the control was $47.2 \pm 5.4$ with no significant difference. The mean duration was $5.7 \pm 4.4$ in group I and $6 \pm 4.5$ in group II, with no significant difference. As regard the presence of retinopathy, peripheral neuropathy, nephropathy and cerebrovascular stroke in both groups there was no significant difference, while there was significant difference between them as regard the presence of ischemic heart disease (IHD) (6\% in group I, $28 \%$ in group II ).

As regard their laboratory investigations (Table 2); there was a significant difference between the patients and control as regard fasting blood sugar, LDL, HDL cholesterol, CRP level, CIMT. So, diabetic patients had higher levels of CRP and CIMT than normal healthy control. We observed that there was no difference between the patients study groups as regard CRP level $(8.18 \pm 3.6$ in group I and $8.5 \pm 4$ in group II) and CIMT (1.2 \pm 0.16 in group I and 1.2 \pm 0.18$)$. 
Table 1: Demographic and Clinical Features of the Studied Groups

\begin{tabular}{|c|c|c|c|c|c|}
\hline & \multicolumn{3}{|c|}{ Study groups } & \multirow[b]{2}{*}{ P-value } & \multirow[b]{2}{*}{ Sig } \\
\hline & $\begin{array}{c}\text { Group I } \\
(\mathrm{n}=50)\end{array}$ & $\begin{array}{c}\text { Group II } \\
(n=50)\end{array}$ & $\begin{array}{c}\text { Group III } \\
(n=50)\end{array}$ & & \\
\hline Male gender & $19(38 \%)$ & $24(48 \%)$ & $25(50 \%)$ & 0.4 & NS \\
\hline Age & $48.3 \pm 6$ & $49.8 \pm 6.2$ & $47.2 \pm 5.4$ & 0.08 & NS \\
\hline Duration of disease(years) & $5.7 \pm 4.4$ & $6 \pm 4.5$ & ------ & & \\
\hline Retinopathy & $22(44 \%)$ & $26(52 \%)$ & ------- & 0.5 & NS \\
\hline Nephropathy & $10(20 \%)$ & $14(28 \%)$ & ------ & 0.4 & NS \\
\hline Neuropathy & $45(90 \%)$ & $42(48 \%)$ & ------ & 0.6 & NS \\
\hline IHD & $3(6 \%)$ & $14(28 \%)$ & ------ & 0.006 & HS \\
\hline CVS & $5(10 \%)$ & $10(20 \%)$ & ------ & 0.1 & NS \\
\hline
\end{tabular}

IHD: ischemic heart disease, CVS: cerebrovascular stroke.

Table 2: Laboratory Investigations and CIMT of the Study Groups

\begin{tabular}{|c|c|c|c|c|c|}
\hline & \multicolumn{3}{|c|}{ Study group } & \multirow[b]{2}{*}{$\mathrm{P}$-value } & \multirow[b]{2}{*}{ Sig. } \\
\hline & $\begin{array}{c}\text { Group I } \\
\text { Mean } \pm \text { SD }\end{array}$ & $\begin{array}{c}\text { Group II } \\
\text { Mean } \pm \text { SD }\end{array}$ & $\begin{array}{l}\text { Group III } \\
\text { Mean } \pm \text { SD }\end{array}$ & & \\
\hline FBS ( mg/dl) & $170.8 \pm 49.1$ & $162.1 \pm 38.4$ & $84.4 \pm 6^{*}$ & $<0.001$ & $\mathrm{HS}$ \\
\hline $2 \mathrm{HPP}(\mathrm{mg} / \mathrm{dl})$ & $283.3 \pm 53$ & $284.5 \pm 52$ & $137.4 \pm 11.2 *$ & $<0.001$ & HS \\
\hline $\mathrm{LDL}(\mathrm{mg} / \mathrm{dl})$ & $53.8 \pm 6.2$ & $54.6 \pm 7.1$ & $62.8 \pm 6.8^{*}$ & $<0.001$ & HS \\
\hline HDL (mg/dl) & $37.4 \pm 2.7$ & $37.2 \pm 2.9$ & $34.6 \pm 1.1 *$ & $<0.001$ & $\mathrm{HS}$ \\
\hline CRP (mg/l) & $8.18 \pm 3.6$ & $8.5 \pm 4$ & $2.7 \pm 0.7 *$ & $<0.001$ & HS \\
\hline CIMT( mm) & $1.2 \pm 0.16$ & $1.2 \pm 0.18$ & $0.5 \pm 0.1 *$ & $<0.001$ & $\mathrm{HS}$ \\
\hline
\end{tabular}

IHD=ischemic heart disease, CVS=cerebrovascular stroke, NS=non-significant, HS=highly significant, FBS=fasting blood sugar, $2 H P P=2$ hour postprandial, $L D L=$ low density lipoprotein, $H D L=$ high density lipoprotein

Table 3 shows the sensitivity and specificity of (LDL), (HDL) and (CRP) in comparison with CIMT as predictors of atherosclerosis. Sensitivity and specificity test for CRP in diagnosis of atherosclerosis disease illustrates probability of being true positive is $(96.3 \%)$ more than being false positive when repeat test 100 times with sensitivity (98\%) and specificity $(72 \%)$.

Table 3: Sensitivity and Specificity of (LDL), (HDL) and (CRP) In Comparison With CIMT

\begin{tabular}{cccc}
\hline Variable & Sensitivity & Specificity & Area under curve (AUC)* \\
\hline LDL & $80 \%$ & $4 \%$ & $18.9 \%$ \\
HDL & $96 \%$ & $36 \%$ & $81.2 \%$ \\
CRP & $98 \%$ & $72 \%$ & $96.3 \%$ \\
\hline
\end{tabular}

Table 4 shows the correlation between CIMT and CRP level among patients groups. It shows that there was strong positive correlation between CIMT and CRP level among group I and group II.

Table 4: Correlation between CIMT and CRP Titre among Study Groups.

\begin{tabular}{cccc}
\hline CRP titre & \multicolumn{2}{c}{ CIMT } \\
\cline { 2 - 4 } & r & p-value & Sig. \\
Group I & 0.5 & 0.001 & HS \\
Group II & 0.6 & 0.001 & HS \\
Group III & 0.3 & 0.07 & NS \\
\hline
\end{tabular}

Table 5 shows the correlation of mean CRP level in all patients with the diabetic complications. Mean CRP level in patients with retinopathy was higher than without retinopathy with a significant difference (9.8 versus7.03 with a P. value $<0.001)$. Mean CRP level in patients with nephropathy was higher than without nephropathy with a significant difference $(10.7$ versus 7.6 with a P. value $<0.001)$. Mean CRP level in patients with IHD was higher than without IHD (10.3 versus 7.9).

Table 5: Correlation between Mean CRP Titre and the Diabetic Complications among All Patients

\begin{tabular}{|c|c|c|c|c|c|}
\hline \multirow{2}{*}{ Complication } & \multicolumn{3}{|c|}{ CRP titre } & \multirow{2}{*}{ p-value } & \multirow{2}{*}{ Sig. } \\
\hline & No. & Mean & SD & & \\
\hline Retinopathy Yes & 48 & 9.8 & 3.3 & \multirow{2}{*}{$<0.001$} & \multirow{2}{*}{ HS } \\
\hline No & 52 & 7.03 & 3.9 & & \\
\hline Nephropathy Yes & 24 & 10.7 & 3.2 & \multirow{2}{*}{$<0.001$} & \multirow{2}{*}{ HS } \\
\hline No & 76 & 7.6 & 3.8 & & \\
\hline Neuropathy Yes & 87 & 8.5 & 3.5 & \multirow{2}{*}{0.3} & \multirow{2}{*}{ NS } \\
\hline No & 13 & 7.3 & 5.7 & & \\
\hline IHD Yes & 17 & 10.3 & 3.2 & \multirow{2}{*}{0.02} & \multirow{2}{*}{$\mathrm{S}$} \\
\hline No & 83 & 7.9 & 3.8 & & \\
\hline
\end{tabular}




\section{Discussion}

Our study aimed to assess CIMT as a predictor of atherosclerosis in T2DM and its correlation to CRP level in these patients. In addition, the study correlated these parameters with LDL-cholesterol levels and the use of statin therapy to evaluate which is more important predictor of atherosclerosis as regard diagnosis and follow up during treatment.

Our results showed that there was significant difference in CRP level between patients groups and the control, with mean CRP level was $8.18 \pm 3.6$ in group I , $8.5 \pm 4$ in group II , and $2.7 \pm 0.7 *$ in group III and the difference was statistically highly significant. Among inflammatory biomarkers, the best evidence to date supports the use of hs-CRP as an independent predictor of increased CVD risk in diabetic and non-diabetic patients. In addition, numerous clinical trials have established elevated CRP levels to be predictive of the development of insulin resistance, metabolic syndrome, and T2DM [7].

Our results are similar to another study [8] which reported that acute phase response was present in patients with type- 2 diabetes. These patients tend to have higher hs-CRP concentrations than control subjects and elevation of hs-CRP in diabetic patients associates with increased risk of non-fatal cardiovascular events and cardiovascular death.

High hs-CRP levels were also found to be strongly correlated and independently with type 2 diabetes: Elevated levels of hs-CRP remained a significant predictor for future diagnosis of metabolic syndrome and diabetes even after adjusting for BMI, family history of diabetes mellitus, smoking, and other factors. Large prospective studies pointed out the involvement of increased hs-CRP on cardiovascular morbidity and mortality in these patients. High levels of hs-CRP have been shown to be an independent predictor of cardiovascular risk for all degrees of severity of metabolic syndrome and T2DM [7], [9], and [10].

Regarding CIMT, there was a significant difference between patient groups and the control with mean CIMT in group I was $1.2 \pm 0.16$, in group-II was $1.2 \pm 0.18$ and in group-III was $0.5 \pm 0.1$. This means that diabetic patients had higher CIMT than healthy control, but there was no difference between both patient groups. Patients with T2DM have a two to fourfold increased incidence of CVD compared to persons without diabetes, which is also reflected by an increased CIMT in T2DM patients [1].

Kotb et al [11] found that CIMT was higher in diabetic patients than in healthy subjects. CIMT, HbA1C, systolic blood pressure, triglycerides, HOMA, and C-reactive protein were significantly higher in patients with uncontrolled than with controlled diabetes.

Naghavi et al. [12] concluded that Patients with CIMT $>1.0 \mathrm{~mm}$, calcium score $>100, \mathrm{CRP}>4 \mathrm{mg} / \mathrm{L}$ and ABI $<0.9$ were classified as at high risk or very high risk for MI, resulting in the recommendations for performing myocardial ischemia test and the target LDL-cholesterol level below $100 \mathrm{mg} / \mathrm{dl}$ or even $<70 \mathrm{mg} / \mathrm{dl}$.

As regard CIMT prediction in T2DM patients; our study showed that LDL had sensitivity $80 \%$ and specificity $4 \%$, HDL had sensitivity $96 \%$ and specificity $36 \%$ while CRP sensitivity was $98 \%$ and specificity was $72 \%$. This means that CRP level was more sensitive and specific as a predictor of carotid atherosclerosis than LDL with considerable difference and more than HDL levels with smaller difference despite that mean LDL in our patients were more or less near normal results in both groups.

Recently XU et al. [13] showed that subjects with CRP $\geq 3 \mathrm{mg} / \mathrm{L}$ were associated with 1.45-fold risk for elevated CIMT. The association was more prominent in subjects with aged $<60$, in diabetic patients and obese subjects. So, they concluded that increased CRP is associated with elevated CIMT in Chinese adults with normal LDL. Low-grade inflammation plays an independent and important role in atherosclerosis.

Tsimikas et al [14] stated that the level of hs-CRP achieved after initiation of statin therapy proved to be of equal importance for subsequent vascular events as was the achieved level of LDL-C. However, the best overall survival was observed among those who not only lowered LDL-C below $70 \mathrm{mg} / \mathrm{dl}$, but who also lowered hs-CRP below $2 \mathrm{mg} / \mathrm{l}$. This effect was present regardless of the statin regimen used.

Our study showed significant strong positive correlation between CIMT and CRP level among group I and group II with $\mathrm{P}<0.001$. These reslts coincide with kotb et al [11] who stated that CIMT were significantly greater in adolescents with T2DM than in healthy subjects and correlated positively with C-reactive protein. They showed a significant elevation of hypersensitive C-reactive protein in uncontrolled than in controlled diabetic patients and this correlated positively with CIMT. Cao et al. [15] showed that CRP concentration was closely correlated with CIMT measures.

Mean CRP in the patients with retinopathy was significantly higher than without retinopathy. Also, other study [16] concluded that CRP levels in patients of retinopathy were elevated as compared to normal controls and diabetics without retinopathy.

Mean CRP in patients with nephropathy was significantly higher than that without nephropathy. Also, in a recent study [17], the authors reported a significant and independent association of CRP level with urinary albumin excretion (UAE) in the microalbuminuric range in patients with T2DM. In a prospective study by Stehouwer et al. [18] included 328 patients with type 2 diabetes followed up for 9 years, found that increased UAE, endothelial dysfunction and chronic inflammation were interrelated processes and the longitudinal development of UAE was significantly and independently determined by such inflammatory markers as hs- CRP and fibrinogen.

We concluded that CRP level is an important parameter of carotid atherosclerosis with good sensitivity and specificity regardless the level of LDL and HDL. Therefore, we can recommend its measurement routinely together with lipid profile either to decide statin use or adjust its dose. 


\section{Acknowledgments}

We are grateful to the consideration and cooperation of all the participants of the study. We thank all patients and healthy persons for participating in this research.

This research did not receive any funding from any organization or company.

\section{References}

[1] L Lundby-Christensen, P Almdal, B Carstensen,et al. Carotid intima-media thickness in individuals with and without type 2 diabetes: a reproducibility study. Cardiovascular Diabetology 2010, 9:40 http://dx.doi.org/10.1186/1475-2840-9-40.

[2] ML Bots, MK Palmer, S Dogan, et al: Intensive lipid lowering may reduce progression of carotid atherosclerosis within 12 months of treatment: the METEOR study. J Intern Med 2009, 265(6):698-707. http://dx.doi.org/10.1111/j.1365-2796.2009.02073.x.

[3] JR Crouse, JS Raichlen, WA Riley, et al. for the METEOR Study Group: Effect of Rosuvastatin on Progression of Carotid Intima-Media Thickness in Low-Risk Individuals with Subclinical Atherosclerosis: The METEOR Trial. JAMA: The Journal of the American Medical Association 2007, 297:1344-1353. http://dx.doi.org/10.1001/jama.297.12.1344.

[4] A Kablak-Ziembicka, T Przewlocki, A Sokołowski,et al. Carotid intima-media thickness, hs-CRP and TNF-_are independently associated with cardiovascular event risk in patients with atherosclerotic occlusive disease. Atherosclerosis 2011; 214: 185-190 http://dx.doi.org/10.1016/j.atherosclerosis.2010.10.017.

[5] L Biasucci, G Biasillo and a Stefanelli. Inflammatory markers, cholesterol and statins: pathophysiological role and clinical importance. Clin Chem Lab Med 2010; 48 (12):1685-1691 http://dx.doi.org/10.1515/CCLM.2010.277.

[6] MG Veller, C Fisher, AN Nicolaides, et al. Measurement for the ultrasonic intima-media complex thickness in normal subjects. $J$ Vasc Surg 1993; 17:719-25 http://dx.doi.org/10.1016/0741-5214 (93)90116-4.

[7] A Pfützner, E Standl, HJ Strotmann, et al. Association of high-sensitive C-reactive protein with advanced stage beta-cell dysfunction and insulin resistance in patients with type 2 diabetes mellitus. Clin Chem Lab Med. 2006; 44 (5):556-60. http://dx.doi.org/10.1515/CCLM.2006.108.

[8] M Soinio, J Marniemi, M Laakso, et al. High sensitivity C-reactive protein and coronary disease mortality in patients with type 2 diabetes. Diabetes Care 2006; 29: 329-33 http://dx.doi.org/10.2337/diacare.29.02.06.dc05-1700.

[9] PM Ridker, JE Buring, NR Cook, and N Rifai. C-reactive protein, the metabolic syndrome, and risk of incident cardiovascular events: an 8year follow-up of 14,719 initially healthy American women. Circulation. 2003; 107 (3):391-7. http://dx.doi.org/10.1161/01.CIR.0000055014.62083.05.

[10] S Devaraj, U Singh, I Jialal. Human C-reactive protein and the metabolic syndrome. Curr Opin Lipidol. 2009; 20(3):182-9. http://dx.doi.org/10.1097/MOL.0b013e32832ac03e.

[11] N Kotb, R Gaber, M Salama, et al. Clinical and biochemical predictors of increased carotid intima-media thickness in overweight and obese adolescents with type 2 diabetes, Diabetes \& Vascular Disease Research 2011.

[12] M Naghavi, E falk, HS Hecht, ET al.SHAPE Task Force. From vulnerable Plaque to vulnerable patient -part III: Executive summary of the screening for heart Attack prevention and Education (SHAPE) task force report. Am J cardiol 2006; 98(2A) 2H-15H http://dx.doi.org/10.1016/j.amjcard.2006.03.002.

[13] M Xu, Y Bi, Y Chen, et al. Increased C- reactive protein Associates with elevated carotid intima -media thickness in chinese adult with normal Low Density lipoprotein cholesterol. Journal of atherosclerosis and thrombosis 2013; 20.

[14] S Tsimikas, J Willerson and P Ridker. C - reactive protein and Other Emerging Blood Biomarkers to Optimize Risk Stratification of Vulnerable Patients. Journal of the American College of Cardiology 2006 Vol. 47, No. 8 Suppl C 19- 31 http://dx.doi.org/10.1016/j.jacc.2005.10.066.

[15] J Cao, C Thach, T Manolio, et al. C - reactive protein, Carotid Intima-Media Thickness, and Incidence of Ischemic Stroke in the Elderly The Cardiovascular Health Study. Circulation 2003. 108:166-170.) http://dx.doi.org/10.1161/01.CIR.0000079160.07364.6A.

[16] S Kaul, RP Morrissey and GA Diamond. Rosuvastatin, inflammation, C-reactive protein, JUPITER, and primary prevention of cardiovascular disease - a perspective. Arch Intern Med 2010; 170:1073-77 http://dx.doi.org/10.1001/archinternmed.2010.189.

[17] VM Patil, S Chaitanyakumar and V Patil. Diabetic nephropathy and its relation to inflammation. International Journal of Pharmacy and Biological Sciences 2013; 3, 117-127.

[18] C Stehouwer, M Gall, J Twisk, et al. Increased urinary albumin excretion, endothelial dysfunction and chronic low grade inflammation in type 2 diabetes: progressive, interrelated and independently associated with risk of death. Diabetes 2002; 51: 1157-1165 http://dx.doi.org/10.2337/diabetes.51.4.1157. 